\title{
Philonsorbonne
}

12 | 2018

Année 2017-2018

\section{Doctorales 2017}

Résumés des Doctorales de Philosophie (2017)

\section{OpenEdition}

\section{Journals}

Édition électronique

URL : https://journals.openedition.org/philonsorbonne/986

DOI : 10.4000/philonsorbonne.986

ISSN : 2270-7336

Éditeur

Publications de la Sorbonne

Édition imprimée

Date de publication : 1 janvier 2018

Pagination : 77-101

ISSN : 1255-183X

\section{Référence électronique}

"Doctorales 2017 », Philonsorbonne [En ligne], 12 | 2018, mis en ligne le 03 mars 2018, consulté le 08 juin 2021. URL : http://journals.openedition.org/philonsorbonne/986 ; DOI : https://doi.org/10.4000/ philonsorbonne.986

(c) Tous droits réservés 


\title{
Doctorales 2017 *
}

\author{
SON ET SENS
}

Jazz et altérité : vers une esthétique de la rencontre

Clément BRESCH

Phénoménologie de l'audible dans l'expérience esthétique

de l'art contemporain depuis le Global Turn

Wilfried BOSSIER

Musique et psychologie chez J. F. Herbart [1776-1841] :

la tâche d'une philosophie du son (Tonpsychologie)

Margaux THURA

Métaphores organiques et mimésis dans les écrits

sur l'architecture de John Ruskin (1819-1900)

Hugues-Antoine NAÏK

\section{AUTEUR ET PERSONNAGE}

L'idiot ET le philosophe chez Jean-Luc Godard

Mauro DE CASTRO

Pour jouer un rôle, l'acteur s'abandonne-t-il à son personnage?

Yoann MALINGE

Le perfectionnisme moral dans le cinéma fantastique

Tristan CHETRIT

\section{LES DONNÉES DE L'EXPÉRIENCE}

Borges et l'ineffable

Florencia Di RocCO

L'expérience intérieure

Raphaël PIERRES

Le statut des données empiriques chez Clarence I. Lewis

Dominik JARCZEWSKI

Contre le mythe du Donné

entre phénoménologie et philosophie analytique

Sergio GENOVESI

\footnotetext{
${ }^{(*)}$. Dans cette rubrique sont publiés les résumés des interventions ayant eu lieu lors des Doctorales de Philosophie organisées à la Sorbonne les 17, 18, 19 et 20 mai 2017, par les doctorants Lyess Bouderbala (CHSPM), Mathieu Frerejouan (PhiCo), Marie Michon (IHPST) et Yu-Jung Sun (GRAmATA).
} 


\section{LANGAGE ET LOGIQUE}

Contradiction et signification dans quelques passages

de la Logique de l'essence de Hegel

Florian RADA

Grammaire et logique dans L'Étoile de la Rédemption

de Franz Rosenzweig

Emeline DURAND

Stanley Cavell et «l'inquiétante étrangeté »:

Contribution à la méthode en psychanalyse

Olivia POIATTI

\section{LA VIE DU SUJET}

l'effet que cela fait de participer à un monde organisé

Victor LEFÈVRE

«La sexualité comme l'intentionnalité sexuelle»:

Un double rapport contradictoire entre le corps et la conscience

Eun-hye $\mathrm{CHOO}$

Nietzsche et le problème de la valeur des évaluations

Eder CORBANEZI

La physique heideggérienne du salut

Matthieu EYCHENIÉ

\section{RAISON ET PASSION}

Rationalité et réciprocité :

perspectives conjuguées de Hobbes, Mauss et Weber

Valérie LOUVEAU

L'Homme-machine :

étude comparée de la conception cartésienne et computationnaliste

Julien BOYER

Lecture comparative de Descartes

et de Malebranche sur la joie des passions

Yeonsik $\mathrm{YoO}$

Les noms divins des choses : platonisme et nominalisme

dans les philosophies de la nature de Schelling et Whitehead 


\section{INDIVIDU ET COLLECTIF}

Max Stirner, C. B. Macpherson et la critique de la propriété

Sebastian KocK

L'utilitarisme et l'idée d'Europe

Ludmilla LORRAIN

L'usage de la notion de vie spinoziste

dans l'ouvre d'Alexandre Matheron

98

Nicolas LEMA HABASH

\section{DROIT ET LOIS}

Lois, mours et opinion publique chez Diderot

Kyosuke TAHARA

La réfutation de l'Empire universel

et l'élaboration du ius gentium chez. Jean Bodin

Shingo АКIMOTO

Le rôle des émotions dans les excuses pénales

Fanny-Elisabeth ROLLER

Vaut-il mieux laisser un coupable en liberté que punir un innocent ?

Nicolas NAYFELD 



\section{SON ET SENS}

\section{Clément BRESCH - Jazz et altérité : vers une esthétique de la rencontre}

Musique d'ensemble parmi tant d'autres, le jazz octroie de fait, dans sa pratique, une place effective à l'autre, à l'alter ego. En d'autres termes, celui-ci donne à voir - et à entendre -, au-delà de la musique effectivement produite et entendue, un certain exercice de la pluralité. Visant à détailler quelque peu ce constat frisant l'évidence, cette intervention cherchera à rendre compte un peu plus clairement et précisément de ce qui, dans le jazz, semble ainsi à l'œuvre en matière d'altérité, sans pour autant verser dans une quelconque ambition par trop particulariste. Pour ce faire, on commencera par s'appuyer sur deux dimensions musicales générales, et on examinera successivement la façon dont celles-ci se déploient concrètement dans la pratique du jazz. On s'intéressera donc, dans un premier temps, à l'horizontalité musicale, c'est-à-dire à la dimension relevant fondamentalement de la mélodie comprise dans son sens le plus simple, que l'on décortiquera progressivement. Puis, dans un second temps, on considérera celle touchant des aspects rythmiques de la musique de jazz, en se concentrant particulièrement sur la notion de swing. Ces deux moments d'analyse nous permettront peu à peu d'esquisser une certaine conception de l'altérité, en œuvre dans la pratique jazzistique collective, concédant - réclamant, même - à l'autre, dans sa pleine individualité, une nette liberté expressive. On pourra à ce stade s'aventurer à ébaucher ce qui pourra constituer un fragment d'une plus générale esthétique du jazz, en donnant à la notion de rencontre une place clef; notion que l'on tâchera d'expliciter et d'articuler avec l'état des lieux alors établi. 


\section{Wilfried BOSSIER - Phénoménologie de l'audible dans l'expérience esthétique de l'art contemporain depuis le Global Turn}

La recherche aborde la question de l'expérience esthétique avec une approche phénoménologique et l'apport de l'histoire de l'art, la critique et la sociologie de l'art, sachant fort bien que l'esthétique est aussi traversée par d'autres approches, comme la sémiologie et la psychanalyse (cf. Jacinto Lageira) et que la notion de l'expérience esthétique est controversée et critiquée, notamment par l'esthétique analytique.

Partant de la pensée de Husserl, Merleau-Ponty et surtout Mikel Dufrenne, cette recherche vise les impensés au sujet du son, de l'expérience de l'audible et du multi-sensoriel (synesthésie), et cela dans le but d'adresser la question centrale de recherche :

Comment analyser le fonctionnement du son, de l'audible et de l'écoute dans l'expérience esthétique de l'art contemporain depuis le Global Turn?

L'objet de recherche, que l'on peut désigner avec Pierre Schaeffer «objet sonore» ou encore «objet esthétique sonore» dans l'art contemporain, recouvre une diversité toujours grandissante de propositions et de pratiques artistiques: sound art, sculptures et installations sonores, musique expérimentale et électroacoustique, video art et installations multimédia, performances...

Le projet partira de l'étude de cas concrets de ces diverses propositions et formes artistiques, i.e. des cas spécifiques, sélectionnés sur la base de leur représentativité et leurs qualités artistiques, dont l'analyse devra permettre d'adresser le questionnement qui découle de la question centrale de recherche: l'Acousmonium (GRM, installation électroacoustique), Christoph De Boeck, Pierre Huyghe, Dominique Gonzalez-Foerster, Janek Schaeffer, Ari Benjamin Meyers, Céleste Boursier-Mougenot, Chantal Akerman...

Pour cerner de façon efficace et critique l'objet de recherche, il faut d'emblée expliciter ce que désigne le terme d'art contemporain. Déjà le terme/l'épithète «contemporain 》 pose problème (cf. Marianne Massin, Nietzsche, Agamben). Dans un deuxième temps, il importe de mettre à jour ce qui rend l'art contemporain distinct de l'art moderne et postmoderne, et, bien entendu, ici les questions de l'autonomie de l'art, de son impact culturel et de sa fonction sociale se posent, comme celles de l'expérimentation artistique et de l'attenante hétérogénéité dans les formes d'art contemporain, ou celle du rôle de la technologie.

L'approche phénoménologique de l'expérience esthétique dans le contexte de la mondialisation invite à en examiner les dimensions culturelles et politiques par le biais de l'histoire de l'art, la critique d'art et la sociologie de l'art, l'esthétique relationnelle de $\mathrm{N}$. Bourriaud et le "partage du sensible » de J. Rancière. 
Margaux THURA - Musique et psychologie chez J. F. Herbart [1776-1841] : la tâche d'une psychologie du son (Tonpsychologie)

Lorsque Johann Friedrich Herbart commence à formuler ses réflexions sur le son et la musique (dès les Psychologische Bemerkungen zur Tonlehre de 1811 que nous prendrons comme point de départ), certaines questions d'esthétique musicale demeurent irrésolues, la théorie acoustique étant encore jugée insatisfaisante; ainsi la question de savoir pourquoi nous jugeons «consonant» un accord parfait ou encore pourquoi nous attachons une représentation différenciée entre les modes mineur et majeur. En effet, si les propriétés physiques du son sont de mieux en mieux connues, l'articulation du phénomène sonore avec nos perceptions et, a fortiori, avec nos représentations non plus seulement sonores mais musicales (un accord, par exemple) nécessitent, selon Herbart, l'éclairage d'une psychologie nouvelle.

Dans un texte tardif que nous pourrons prendre comme point d'arrivée, les Psychologische Untersuchungen de 1839, Herbart, en «kantianer Verfasser $\gg$ (auteur kantien) semble donner à la psychologie une assise qu'il voudrait entre l'empirisme et la métaphysique. En effet, selon lui, la théorie psychologique ne devrait jamais prétendre pouvoir se passer de l'expérience, mais cette expérience devra être elle-même constituée de telle sorte que la théorie (Lehre) puisse s'y appliquer. En ce qui concerne l'expérience musicale qui nous intéresse, nous verrons qu'elle devra tout d'abord être réduite à ses éléments, à ses plus petits composants possibles, pour pouvoir être débarrassée de certaines conceptions peu fiables et peu précises qui feraient obstacles à une génération psychologique de l'harmonie musicale: aussi, nous verrons, entre autres, quel pourrait être le gain théorique à détacher l'idée de dissonance de celle d'une gêne auditive (d'une impression de battement ou de frottement: zittern, schwirren,...) par exemple, ou encore à caractériser l'octave, tenue pour la consonance la plus parfaite dans les théories classiques, comme l'intervalle musical contenant le plus fort degré d'opposition (Gegensatz) entre ses deux sons.

\section{Hugues-Antoine NAÏK - Métaphores organiques et mimésis dans les écrits sur l'architecture de John Ruskin (1819-1900)}

Qu'est ce qu'une forme organique en architecture ? Il est bien entendu que les bâtiments ne sont pas des êtres vivants, et pourtant, l'usage de métaphores organiques pour les qualifier est extrêmement répandue, et ce au moins depuis le $19^{\mathrm{e}}$ siècle. Mais quelle est la signification de cette métaphore? Que veut-on dire lorsque l'on compare un bâtiment à une plante, ou à un organisme en croissance ?

Pour trouver une réponse, j'entends reposer la question sur le plan esthétique. Il ne s'agit pas en effet ici d'établir un catalogue des diverses significations de la métaphore organique appliquée à l'architecture (ce qui a par ailleurs déjà été fait), mais d'en interroger le sens esthétique : que 
signifie la référence au vivant lorsqu'il s'agit de décrire les formes de l'architecture, et l'émotion que celle-ci peut susciter ? L'enjeu consiste ici à donner un sens esthétique au terme de vie, par l'étude d'un art a priori non figuratif, pour lequel prendre l'apparence de la vie ne signifie justement pas représenter le vivant.

$\mathrm{Au}$ cours de cette intervention, je ferai ainsi l'hypothèse que l'usage esthétique de la métaphore organique en architecture désigne en réalité un cas particulier de mimésis dans lequel l'objet de l'imitation est sans doute moins le vivant au sens biologique que le psychisme lui-même, qui se retrouve "objectivé » dans l'architecture par un processus de projection. Sous les apparences du biomimétisme, c'est ainsi de psychomimétisme qu'il serait question.

Je tenterai de le montrer ici à travers l'étude de l'œuvre de John Ruskin (1819-1900), dont les écrits définissent ce qu'est une forme organique en architecture à travers une réflexion sur le style gothique. On verra ainsi que ce qui se joue là c'est bien la transposition à l'architecture d'une sensibilité au vivant, qui conduit à accorder une importance toute particulière à sa matérialité.

\section{AUTEUR ET PERSONNAGE}

\section{Mauro DE CASTRo - L'idiot ET le philosophe chez Jean-Luc Godard}

Jean-Luc Godard aime «faire l'idiot». Personnage récurrent dans la littérature, le théâtre et le cinéma, l'idiot exerce souvent une fonction comique, mais aussi ironique, il crée un contraste avec une réalité donnée et met en cause ses présupposés. Gilles Deleuze va jusqu'à lui attribuer une fonction philosophique, comme celui qui pousse la pensée à sa propre limite, vers l'impensable, raison pour laquelle chez certains auteurs l'idiot possède une constitution hybride, entre figure esthétique («puissance d'affects et de percepts») et personnage conceptuel («puissance de concepts»). De Socrate à Descartes, de Dostoïevski à Melville, de Kurosawa à Godard, l'idiotie ne s'identifie pas à l'imbécillité ; l'idiot ne s'oppose pas au sage, mais à la prétention du savoir. Faire l'idiot est un exercice de pensée. L'idiot chez Godard peut être interprété dans cette même direction dès ses premiers

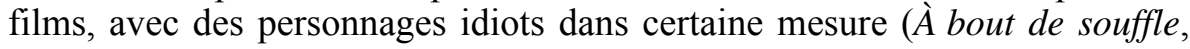
Les carabiniers...) qui problématisent la morale, la politique ou l'existence, mais surtout dans les films où Godard lui-même joue l'idiot et assume son rôle double de «cinéaste-idiot» (Prénom Carmen, Soigne ta droite, King Lear...), en problématisant la réalisation et la production cinématographiques. On peut dire que l'idiot personnifié par Godard 
(acteur/réalisateur) est une sorte de mise en abîme, il traduit la place paradoxale de Godard dans l'histoire du cinéma; à mesure qu'il prétend atteindre la limite de la représentation et du langage cinématographique, il devient un cinéaste à la fois célèbre et méconnu, vénéré et honni. Tel le personnage de Dostoïevski (revisité dans Soigne ta droite), Godard incarne à la fois le Prince et l'idiot du cinéma. D'ailleurs, il nous semble que l'idiot doit être mis en rapport avec un autre personnage qui traverse l'œuvre de Godard, celui du philosophe (Vivre sa vie, La Chinoise...), de telle sorte que le binôme "idiot-philosophe» nous permet d'entrevoir chez Godard une certaine conception du philosopher. Ainsi, on pourrait dire encore que Godard aime « faire le philosophe ». Il s'agit donc d'analyser les puissances esthétiques de la figure de l'idiot chez Godard et d'en discuter les enjeux philosophiques.

\section{Yoann MALINGE - Pour jouer un rôle, l'acteur s'abandonne-t- il à son personnage?}

Qu'il soit envisagé comme une interprétation ou comme un jeu, le rapport de l'acteur à son rôle est problématique. Cette communication s'intéresse au rapport qu'un être humain entretient avec un ensemble de significations qu'il doit mettre en œuvre sur une scène, ensemble qui comprend à la fois des paroles et des mouvements (gestes et expressions de sentiments).

Or ce rapport ressemble à celui que les êtres humains entretiennent avec ce qu'ils disent et font dans la réalité. Toutefois, il s'en distingue de deux manières au moins : $1^{\circ}$ il est défini par avance (le rôle) ; $2^{\circ}$ il est encadré dans un espace clos, celui de la scène, espace qui se superpose à la réalité dans laquelle nous vivons.

Il faut donc se demander quel rapport entretient l'acteur à cet ensemble de significations: est-ce qu'il s'approprie l'ensemble «paroles et mouvements » au point d'en devenir l'agent ou bien l'acteur ne sait-il pas toujours que le rôle lui est extérieur? Alors ne se considère-t-il pas comme un simple vecteur instrumental permettant au processus abstrait écrit i.e. son rôle - de devenir concret ? Cette antinomie que nous pourrions synthétiser par l'opposition de l'abandon et du contrôle de l'acteur a fait l'objet d'un débat philosophique historique, dont on présente d'abord de part et d'autre deux figures, celle de Pierre Nicole et celle de Diderot.

Cependant, la finalité de cette communication est de montrer que Sartre, dans plusieurs textes, est l'héritier de ce débat et y prend part d'une manière nouvelle en insistant sur le statut ontologique du rôle comme analogon. Nous cherchons à montrer que par là, il dépasse l'antinomie classique. En mettant la théorie sartrienne en contraste avec la formation de l'acteur pensée par Stanislavski, nous cherchons à comprendre que le rapport de l'acteur à son rôle engage une compréhension philosophique de la subjectivité. Chez Sartre, l'acteur pourrait-il alors apparaître comme l'incarnation du rapport de tout être humain à sa propre condition? 


\section{Tristan Chetrit - Le perfectionnisme moral dans le cinéma fantastique}

Le perfectionnisme moral diffère de la plupart des autres théories morales par la place capitale qu'il accorde aux devoirs envers soi-même. Il souligne l'importance pour l'individu de trouver des biens objectifs dans sa propre vie et de développer ses aptitudes. Contrairement à d'autres théories, il n'accorde pas uniquement de l'importance aux actes envers autrui.

Le perfectionnisme moral que développe R.W. Emerson (notamment dans Self Reliance [La confiance en soi]) et celui présent dans la philosophie de Nietzsche ou encore celui de Stanley Cavell semblent particulièrement appropriés pour analyser certains personnages de films fantastiques que l'on peut regrouper sous l'appellation de princes monstres.

Cette dénomination correspond à un concept original qui est transversal aux classifications traditionnelles des monstres (vampires, fantômes...). Elle rassemble des personnages hors normes, premiers dans leur domaine ou pour quelqu'un, torturés ou en marge de la société et présentant des pouvoirs surhumains ou jouissant d'une grande influence. On peut considérer la Bête dans le film La Belle et la Bête de Jean Cocteau comme un archétype de ces personnages. Le transcendantalisme à travers les idées de confiance en soi, d'aversion du conformisme, de retrait de la société tout comme les concepts nietzschéens de volonté de puissance ou d'éternel retour se révèlent pertinents dans l'étude de ces caractères.

Les princes monstres mettent en lumière nos ambivalences, reflètent nos aspirations à la grandeur et à la différence et nous interpellent sur notre rapport à la mémoire dans les sociétés modernes. L'analyse du perfectionnisme de ces personnages permet de comprendre à la fois l'importance de cette théorie philosophique et ses ramifications dans une partie de la culture populaire à travers des œuvres du septième art.

\section{LES DONNÉES DE L’EXPÉRIENCE}

\section{Florencia DI RocCo - Borges et l'ineffable}

Parmi les «versions borgésiennes» de la thèse de l'« ineffabilité de l'expérience » les plus commentées, on trouve celles qui s'appliquent d'avantage à l'expérience "privée», ou encore à celle du «texte». Ce n'est que dans les années quarante, anticipant les premières ébauches de la " cybernétique », que Borges en présentera une version littéraire et non plus essayistique, s'appliquant exclusivement à l'«expérience sensible». C'est Funes el memorioso. 
Rappelons que l'argument de l'ineffabilité de l'expérience sensible, alternativement appelé « de la finesse de grain » (Evans, Peacocke) suppose que nous pouvons opérer des discriminations perceptives beaucoup plus fines que les discriminations autorisées par les concepts observationnels nous possédons, au mieux, une centaine de noms pour la couleur « vert», alors que nous pouvons en distinguer un nombre indéfini.

$\mathrm{Si}$ cette thèse n'est jamais énoncée au cours de la nouvelle, la construction de l' " hypermnésie » de Funès repose sur l'idée selon laquelle la mémoire ordinaire - rudimentaire, grossière - est un mode de stockage à la mesure des concepts du langage ordinaire. Quelqu'un avec une mémoire parfaite devrait mettre en évidence l'insuffisance des concepts ordinaires à rendre le réel dans toute sa netteté.

L'objectif de cette intervention est de lire la nouvelle à la lumière de la formulation évanesienne de l'argument, tout en montrant comment la logique des «langages phénoménologiques» inventés par Funès suit les prémisses de la réponse «constructiviste» modowellienne, celle des dits «concepts déictiques». L'analyse de la fiction permettra de pointer, toutefois, l'un des problèmes de "grammaire » de cette solution, dont l'explicitation des présupposés nous aidera à «relativiser", à son tour, l'argument évanesien.

\section{Raphaël PIERRES - L'expérience intérieure}

L'enjeu de cette communication est d'interroger l'expérience intérieure en ce qu'elle a de plus problématique: une expérience au plein sens du terme est-elle concevable sans une forme d'extériorité entre l'observateur et ce qu'il observe, entre spectateur et spectacle ? J'ai ainsi souhaité me saisir de ces Journées Doctorales comme d'une occasion d'ouvrir un dialogue entre histoire de la philosophie et approches contemporaines autour d'une notion transversale problématique.

Pour ce faire, je commencerai par manifester la fécondité d'une mise en perspective historique en prenant pour point de départ le commentaire des propositions décisives permettant de comprendre la réflexion comme expérience intérieure au cœur de l'Essay on Human Understanding : Locke nous y invite à considérer la réflexion comme perception des opérations mentales, et, en premier lieu, perception de perception. L'expérience interne devrait en ce sens être saisie comme une perception au second degré.

Il est pourtant contestable d'une part que l'esprit puisse se contempler en train d'agir, si la contemplation s'oppose à l'activité ; d'autre part que l'observation puisse être considérée elle-même comme active, sans risque de modifier ce qu'elle observe. Ainsi prise en tenaille, l'expérience intérieure présente un caractère instable, évanescent, qui ne semble plus lui permettre de fonder une connaissance. Cette critique nous rendra lisible la manière dont les débats sur la possibilité de faire de la psychologie une science à la fin du $19^{\mathrm{e}}$ siècle remettent en jeu le statut épistémologique de l'introspection. 
Nous en tirerons toutes les conséquences à la lecture des pointes contre l'expérience intérieure lancées par Wittgenstein, incisives à plus d'un titre, et peut-être en raison-même de leur ambivalence, de la pluralité de leurs interprétations et applications possibles. Cela nous permettra enfin de proposer, à travers une relecture infidèle de Bataille, une ouverture vers la dimension esthétique de ce problème de l'expérience intérieure, permettant en particulier d'interroger à la fois son caractère historique, et, en regard, certaines formes littéraires contemporaines.

\section{Dominik JARCZEWSKI - Le statut des données empiriques chez Clarence I. Lewis}

Dès ses débuts, la philosophie de Clarence Irving Lewis, le pragmatiste américain méconnu non seulement en France, mais aussi dans le monde anglo-saxon, vise à surpasser les oppositions marquant la théorie de la connaissance de la fin du $19^{\mathrm{e}}$ et de la première partie du $20^{\mathrm{e}}$ siècle. Dans mon intervention, je me concentre sur sa théorie des données empiriques, un des sujets centraux pour la philosophie de la connaissance, de l'esprit et, dans les dernières décennies, l'objet de considération de sciences cognitives. La position du représentant de la deuxième génération du pragmatisme, en attribuant aux qualités le privé sans exception, pratiquement les élimine du domaine de la connaissance. En même temps, le philosophe défend l'irréductibilité des qualia, nom dont il est l'auteur. Il critique le mythe de données avant lettre et il fonde la signification de langue sur les sensations, aussi dans le cas des termes universaux et les propositions analytiques. La reconstruction de sa philosophie que je présente n'est pas seulement une position intéressante dans le contexte contemporain, mais elle aide aussi à mieux comprendre la carte de théories concurrentes de données empiriques et les distinctions qui ne sont pas toujours visibles. Dans ma conclusion, je propose de renouveler l'approche à la question du cadre de la théorie de l'expérience immédiate. Je montre dans quel sens cette interprétation aide à intégrer les éléments divers de la théorie lewisienne et pourquoi, bien comprise, elle offre beaucoup plus que ce que l'on attend d'une curiosité historique comme on la considère souvent.

\section{Sergio Genovesi - Contre le Mythe du Donné entre phénoménologie et philosophie analytique}

Le «mythe du donné » est le nom que Sellars a attribué, dans son essai Empiricism and Philosophy of Mind, à toute philosophie qui cherche à fonder notre représentation du monde, et l'objectivité en général, sur des données extérieures qui constitueraient le premier commencement absolu de notre connaissance. Ainsi, Sellars et McDowell rejettent cette idée fondationnaliste et argumentent en faveur de l'impossibilité de séparer notre conceptualité des données sensibles, ce que suggère le titre même du livre de McDowell : Mind and World. Une telle réflexion autour de la question 
du donné et du fondationnalisme est également présente chez différents phénoménologues français. Le mythe du donné est, en fait, un autre nom que l'on donne à la question, très débattue, de l'origine de la connaissance empirique et du rapport entre les données objectives et les sujets. On peut penser, par exemple, au concept de supplément d'origine introduit par Jacques Derrida ou à la phénoménologie de la donation élaborée par JeanLuc Marion, ainsi qu'à la théorie du holisme de l'expérience développée récemment par Claude Romano.

L'intervention articulera une confrontation entre les principales critiques adressées au fondationnalisme, et les solutions alternatives qui ont été proposées, soit du côté analytique soit du côté phénoménologique. Autrement dit, nous chercherons, donc, d'un côté à dégager les alternatives au mythe du donné les plus pertinentes et, d'un autre côté, à mettre en évidence les difficultés théoriques que soulèvent les positions nonfondationnalistes. Le but de cette confrontation sera d'établir si les alternatives envisageant la dissolution de cette division donné/donataire, c'est-à-dire entre objectivité et subjectivité, parviennent vraiment à échapper aux paradoxes qui touchent le fondationnalisme ou si elles introduisent plutôt de nouveaux paradoxes, courant le risque, par ce fait, de retomber dans une autre fondation mythique.

\section{LANGAGE ET LOGIQUE}

\section{Florian RADA - Contradiction et signification dans quelques passages de la Logique de l'essence de Hegel}

Cette intervention prend pour point de départ une question kantienne qui constitue le nerf de la déduction des catégories et de l'analytique des principes : comment les catégories peuvent-elles avoir un «sens et une signification ", c'est-à-dire s'appliquer aux données de la sensibilité ? La réponse kantienne, brièvement rappelée, pourrait être que c'est par la conjonction de la spontanéité de l'entendement et de la réceptivité de la sensibilité, conjonction rendue possible par la faculté de juger, que les catégories peuvent avoir un contenu. Mais la rencontre la spontanéité et de la réceptivité reste marquée par une hétérogénéité irréductible de ces facultés. La «chose en soi » apparaît ainsi comme un rappel de cette irréductibilité. La lecture hégélienne d'une telle différence, dès Foi et savoir, met au premier plan la structure du jugement comme articulation du sujet (le particulier, dans la forme de l'être) et du prédicat (l'universel, dans la forme de la pensée). Ce serait alors dans une thématisation trop rapide de la fonction de la copule, articulant désormais le donné et l'universel, qu'il faudrait chercher la source de la différence entre entendement et sensibilité : 
si la copule énonce une identité, alors le seul jugement rigoureux est tautologique, mais si elle énonce une différence, alors on prend le risque de l'indétermination du jugement ou du non-sens (puisque n'importe quelle chose différente du sujet pourrait être dite du sujet). Notre proposition consiste à lire certains passages des Déterminations de la réflexion, dans la Logique de l'essence et du Jugement dans la Logique du concept afin de reconstituer la théorie hégélienne de la copule (au sein de la progression identité - différence - contradiction). Celle-ci produirait un mouvement d'explicitation du sujet (corrélat d'une désignation) dans son prédicat (corrélat d'une définition). Nous voudrions ainsi montrer que la copule n'est pas une simple identité entre deux «termes» qui laisseraient supposer l'autonomie de ce dont ils tiennent lieu, mais une circulation entre deux opérations du langage: parler de quelque chose (désignation) et en dire quelque chose (définition).

\section{Emeline DURAND - Grammaire et logique dans L'Étoile de la Rédemption de Franz Rosenzweig}

Dans son grand œuvre L'Étoile de la Rédemption (1921), Franz Rosenzweig installe le souci du langage au cœur de son projet philosophique. Il s'agit de refonder la philosophie comme «pensée parlante » ou pensée du langage (Sprachdenken), et de s'appuyer sur les faits du langage pour en retirer des enseignements épistémologiques, existentiels, théologiques.

C'est dans ce cadre que Rosenzweig met en place une opposition nette entre la logique et la grammaire. Voyant dans l'idéalisme hégélien un panlogisme, Rosenzweig lui reproche de s'appuyer sur une logique « ennemie de la langue », dont les lois propres démentent celles du langage. L'idéalisme tend ainsi à se détourner de toute philosophie du langage, mais aussi de toute écoute du texte sacré, isolant l'une de l'autre la philosophie et la Révélation. Dans la démarche de Rosenzweig, la grammaire vient faire contrepoids à ce refus du langage, en rapprenant aux philosophes à écouter et à répondre. L'« analyse grammaticale » devient le nom d'une forme d'exégèse consistant à déchiffrer la Révélation, phénomène fondamental de la parole humaine et divine, dans le trésor originel des formes linguistiques bibliques.

Pourtant, l'opposition entre logique et grammaire doit sans doute être relativisée. Rosenzweig suggère que la logique et la grammaire, loin de représenter deux attitudes radicalement opposées face au langage, constituent deux aspects du langage lui-même, unis par une relation de promesse. C'est cette relation qu'il faut élucider pour montrer en quoi Rosenzweig propose une contribution originale à la philosophie du langage qui, tout à la fois, dénonce les apories de l'idéalisme, reprend les intuitions grammairiennes de Humboldt et Rosenstock-Huessy, et pose, peu de temps avant Wittgenstein et dans des termes tout différents, la question d'une grammaire philosophique. 


\section{Olivia PointTi - Stanley Cavell et "l'inquiétante étrangeté 》: contribution à la méthode en psychanalyse}

Stanley Cavell souligne à de nombreuses reprises dans son œuvre la parenté en termes de visée "thérapeutique » entre la philosophie et la psychanalyse. Il indique en particulier les liens méthodologiques entre l'œuvre de Wittgenstein et celle de Freud.

Cette méthode commune, Cavell en parle parfois comme d'une « inquiétante étrangeté », reprenant le concept freudien, que l'on peut comprendre comme l'irruption dans le langage d'un «familier» qu'on ne reconnait plus.

Il est possible d'étendre cette parenté entre philosophie analytique et psychanalyse en insistant sur le pari que font l'une et l'autre de revenir au langage ordinaire et de lui rendre sa densité. Ce point peut être illustré par le travail, entre autres, de Cavell sur l'œuvre de Beckett, qui, en rendant aux mots leur sens littéral, nous permet de les entendre différemment.

Cette parenté méthodologique n'est cependant pas tout à fait explicitée par Stanley Cavell, et mériterait me semble-t-il d'être approfondie. Ce point pourrait nous permettre d'expliquer comment, par quel moyen, la psychanalyse amène ses analysants à voir le réel.

Il reste cependant une différence majeure dans cette méthode commune, que Cavell ne mentionne pas, et qui peut être décrite comme la prise en compte par la psychanalyse de la difficulté terrible qu'il peut y avoir à mettre en œuvre cette méthode, difficulté qui n'est pas, et c'est peut-être là l'apport de la psychanalyse, d'ordre moral.

Je propose donc d'explorer les liens entre philosophie du langage ordinaire et psychanalyse, dans la mesure où elles prétendent toutes deux à une forme de guérison, en explorant d'une part leur proximité méthodologique et d'autre part leurs divergences quant aux présupposés d'application de cette méthode.

\section{LA VIE DU SUJET}

\section{Victor LEFÈVRE - L'effet que cela fait de participer à un monde organisé}

Dans notre thèse de doctorat, nous défendons que les écosystèmes sont des êtres organisés, au sens que Kant donne à cette expression dans la Critique de la Faculté de Juger, c'est-à-dire des totalités dont les parties dépendent les unes les autres pour l'existence. Les parties en question sont 
autant des entités non vivantes que des organismes. Parmi ces derniers se trouvent des sujets, et l'objet de notre communication est d'examiner ce qu'implique la thèse du caractère organisé des écosystèmes du point de vue de ces vivants particuliers. Quel effet cela fait-il pour un vivant doué de conscience de constituer son milieu d'une manière telle qu'il est engagé dans une dépendance mutuelle avec les autres constituants de ce milieu ? Quel sens y a-t-il à inscrire son existence dans un monde ainsi co-produit ?

\section{Eun-hye Cноо - «La sexualité comme l'intentionnalité sexuelle ": un double rapport contradictoire entre le corps et la conscience}

En mettant en lumière l'analyse phénoménologique de la sexualité chez M. Merleau-Ponty, cette étude permet une compréhension de la structure existentielle de la sexualité en tant qu'expérience vécue pour nous. La sexualité ne peut être réduite au mécanisme physiologique ou à l'acquisition culturelle, soit la dimension du corps, soit celle de la conscience ; la plus simple définition de la sexualité est «différence sexuelle»; en termes phénoménologiques, cela signifie la différence de la manière intentionnelle de vie. On peut renvoyer à ce que Merleau-Ponty affirme de son propre projet phénoménologique en recherchant une intentionnalité originale; surtout, il trouve le fil conducteur de ce projet à partir de la vie sexuelle, de telle sorte que la sexualité dévoile l'intentionnalité sexuelle à partir de laquelle on peut s'orienter vers l'autre, ou encore désirer l'autre. De là, il suit que trois questions de l'intentionnalité sexuelle sont pertinentes pour mon propos. Dans un premier temps, à partir de quoi on parvient à la différence de la manière intentionnelle. La réponse est qu'il en va du mode de corrélation entre le corps propre et la base de l'expérience perceptive. Dans un deuxième temps, concernant la caractérisation du corps propre, de quel point de vue faut-il se placer pour éclaircir son phénomène sexuel ? Une fois ce point de sexualité mis au jour, qu'est-ce que le corps humain qui n'est pas seulement une chose au même titre que les étants mondains, ni un certain résultat de la spiritualisation?

Finalement, par comparaison avec l'intentionnalité pulsionnelle de Husserl, nous posons la question de savoir si l'intentionnalité sexuelle implique une téléologie universelle à la hauteur de la conscience transcendantale.

\section{Eder CORBANEzI - Nietzsche et le problème de la valeur des évaluations}

Dans cette étude, nous comptons défendre que la relation entre les notions de vie, de valeur et de volonté de puissance constitue une condition nécessaire pour la procédure nietzschéenne destinée à apprécier la valeur des évaluations, c'est-à-dire à évaluer et à hiérarchiser les évaluations. Nous commençons par analyser la manière par laquelle Nietzsche établit une 
association intrinsèque des notions de vie, de valeur et de volonté de puissance : selon lui, vivre consiste à évaluer, et toute évaluation traduit à son tour l'exercice de la volonté de puissance. Ensuite, en nous interrogeant sur la relation complexe entre les évaluations et la vie, nous espérons faire ressortir que, d'une part, les évaluations se révèlent être une condition nécessaire pour toute forme de vie, puisqu'il est impossible de vivre sans évaluer, mais d'autre part, résultant d'interprétations, les évaluations peuvent jouer le rôle opposé, à savoir conduire au dépérissement. En tout cas, soit condition d'existence, soit facteur de dépérissement, les évaluations sont considérées par Nietzsche comme des symptômes de formes de vie. Et c'est justement la logique de cette démarche d'évaluation des évaluations qu'il faut alors prendre pour objet d'analyse. Ce faisant, nous essayons de montrer que, aux yeux de Nietzsche, toute évaluation configure l'affirmation d'une forme de vie et par conséquent la négation d'autres; bien plus : à la limite, toute évaluation équivaut à un jugement sur la valeur de la vie elle-même. Et pourtant, puisque les jugements sur la valeur de la vie sont nécessairement injustes, en raison de leur caractère interprétatif, ils n'ont de valeur qu'en tant que symptômes d'une vie ascendante ou d'une vie décadente. Notre étude aboutit à la mise en évidence de la fonction que joue à l'intérieur de cette démarche la conception nietzschéenne de vie comme volonté de puissance, conception qui elle aussi résulte d'une interprétation et comporte des évaluations.

\section{Mathieu EYCHENIÉ - La physique heideggérienne du salut}

Dans les paragraphes 54-62 d'Être et temps, Heidegger tente de rendre compte de la possibilité du dévoilement de l'authenticité (Eigentlichkeit) du Dasein. Cette mise au jour nécessite un mouvement particulier, une «modification existentielle du On» (Etre et temps, trad. E. Martineau, édition numérique hors-commerce, p. 212) : la compréhension de l'appel de la conscience (le vouloir-avoir-conscience), qui ouvre sur la résolution devançante (vorlaufende Entschlossenheit). Dans Être et temps, Heidegger développe ainsi ce que l'on peut appeler une physique du salut. L'enjeu principal de notre travail sera de déterminer les sources de cette physique. Nous nous concentrerons, outre les paragraphes d'Être et temps déjà évoqués, sur deux séries de textes : les cours consacrés à Aristote au début des années 1920 d'un côté (en particulier le cours de 1924-1925, qui débute par une étude des cinq modes de l'alètheuein mentionnés au livre VI de l'Éthique à Nicomaque), le cours de 1920-1921, portant sur saint Paul, ainsi que la conférence de 1924, intitulée "Le problème du péché chez Luther », de l'autre. Nous tenterons de montrer que le mouvement vers le salut est compris comme une " intériorisation » du Dasein, en un double sens : en un sens aristotélicien d'une part (sur la base de l'analyse de la phronèsis aristotélicienne, interprétée comme conscience inamissible du Dasein, Heidegger considère la modification existentielle du On comme une hexis, partant comme une forme déterminée d' «être dans »), en un sens luthérien 
d'autre part (l'authenticité heideggérienne correspond, chez Luther, à la vie selon l'"homme intérieur», considéré non comme une composante de l'homme ou "un "habitus" vide " (ibid., p. 234) en lui, mais comme une tendance de l'existence, qui ne conduit pas à la suppression de la tendance contraire, selon la thèse de l'homo simul justus et peccator.

\section{RAISON ET PASSION}

\section{Valérie LOUVEAU - Rationalité et réciprocité : perspectives conjuguées de Hobbes, Mauss et Weber}

La promesse s'envisage comme une interaction sociale au cours de laquelle promettant et destinataire de la promesse procèdent à un échange de dons maussien, la parole contre la confiance. Cet échange de dons actualise la transmission d'un fidéicommis : la confiance en la bonne foi du promettant, bonne foi qui vise la préservation des intérêts d'autrui. Ainsi, la promesse acceptée instaure une relation de confiance structurée dans la durée par la réciprocité.

Quelles sont les raisons qui conduisent les individus à s'engager durablement dans une telle relation? À partir de l'approche hobbesienne de la promesse, nous montrerons que la réciprocité articule chez l'individu rationalité en finalité et rationalité en valeur, types de rationalité mis au jour par Weber.

La mise en évidence des dimensions rationnelles de la réciprocité nous permettra alors de travailler la notion wébérienne de rationalisation à la lumière de ce plan de la socialité. Quelle «place» la réciprocité prend-elle par rapport au processus de rationalisation de la vie sociale? Nous examinerons cette hypothèse: puisque la réciprocité nous porte au plan archaïque, toujours sensible au présent, de la socialité, peut-on dire qu'elle fait partie du processus de rationalisation, qu'elle peut déborder; et, par suite, que le processus de rationalisation ne se définit pas seulement comme une tendance de l'activité sociale à s'orienter de manière rationnelle en finalité ?

On signalera, sans entrer dans le détail ici, que ce travail sur les rapports qu'entretiennent réciprocité et rationalisation vise à mieux comprendre la dynamique de la Responsabilité Sociale de l'Entreprise (RSE). En effet, le mouvement de la RSE, qui résulte depuis son émergence d'initiatives prises par les entreprises qui comprennent les attentes sociales, tend aujourd'hui à une rationalisation qui affirme les relations entre le droit et la RSE. 


\section{Damien LACROUX - L'Homme-machine : étude comparée de la conception cartésienne et computationnaliste}

Le Traité du Monde, incluant le chapitre portant sur L'Homme, est presque achevé en 1633 et c'est en particulier dans ce chapitre que Descartes développe une épistémologie du corps humain fondée sur le modèle de la machine. La notion de machine au sens cartésien du terme perdurera tant dans l'histoire de la philosophie que dans l'histoire des sciences. Malgré les avancées remarquables de la cybernétique au $20^{\mathrm{e}}$ siècle, le modèle cartésien semble inébranlable et cela, au point que certains penseurs voient dans la machine cartésienne les éléments fondamentaux de ce qui deviendra par la suite la machine cybernétique homéostatique. C'est véritablement à partir de 1936 qu'un nouveau modèle machinique émerge comme radicalement différent du modèle cartésien. Ce modèle, appelé machine logique, a été élaboré par Alan Turing à des fins militaires pour contrer la redoutable machine de codage SIGMA du troisième Reich. La postérité fera de la machine logique le fondement théorique de nos ordinateurs ainsi que le terreau où se développera une nouvelle discipline informatique : l'intelligence artificielle. Enfin, la machine logique inspirera les courants de pensée computationnalistes selon lesquels l'Homme est réductible, tant dans son fonctionnement neurobiologique que neuropsychologique, à une machine. Le computationnalisme opère donc un retour à la conception cartésienne de l'Homme-machine sans pour autant s'appuyer sur le même modèle machinique. Quelles sont alors les principales différences qui séparent le concept cartésien et le concept turingien de machine ? Cette rupture dans la façon de penser la machine est-elle véritablement fondée ou bien existe-t-il encore une continuité entre la machine cartésienne et la machine formelle? Enfin, le computationnalisme répète-t-il à l'identique mais avec un modèle différent la tentative cartésienne de penser l'Homme sur le modèle de la machine, ou bien va-t-il plus loin que son prédécesseur cartésien dans ce projet épistémologique?

\section{Yeonsik Yoo - Lecture comparative de Descartes et de Malebranche sur la joie des passions}

Dans ma communication, je voudrais vous présenter une réflexion sur la joie de s'émouvoir de toutes les sortes de passions (l'amour, la haine, la joie, la tristesse, etc.) à travers la lecture comparative du Traité des passions de Descartes et de Malebranche.

Chez Descartes, cette joie est le concept qui termine son traité physique des passions. Elle constitue un souverain remède contre les passions. Elle peut être acquise par l'exercice de la vertu. Dans ce sens, elle est une connaissance présupposée pour la morale.

Dans ce traité, cette joie s'explique par l'exemple du plaisir littéraire et théâtral des passions. Ce plaisir s'éclaircit par analogie avec la genèse physique du plaisir du corps. Ce plaisir n'est pas une passion qui se rapporte 
au bien (la force ou la bonne disposition) du corps. Il est une action vertueuse qui concerne le bien de l'âme (un mouvement de la volonté sur la force de l'âme). Cette action volontaire de la bonne disposition de l'âme s'appelle autrement joie intellectuelle, agréable émotion intérieure ou pensée raisonnable.

Dans le Traité méthodologique des passions de Malebranche, la joie des passions n'est pas non plus une passion des passions, mais elle est un sentiment immoral du corps. Ce sentiment sensible accompagne toutes les passions qui sont causées par la réaction nécessaire du corps, pour son bien, face à des objets extérieurs. Cette bonne disposition corporelle des passions cause ce sentiment joyeux qui rend toutes les passions agréables et nous esclaves des biens des corps.

Pour finir je souhaite vous proposer de discuter sur ces questions. La passion de tristesse est-elle joyeuse, seulement à condition que nous ayons la force de la supporter ou de la maîtriser. Ou bien la passion de tristesse estelle toujours agréable? En ce sens, nous oblige-t-elle à continuellement rester à elle?

\section{Louis MORELLE - Les noms divins des choses : platonisme et nominalisme dans les philosophies de la nature de Schelling et Whitehead}

Le projet moderne d'une philosophie de la Nature peut se trouver adéquatement résumé dans l'exclamation lautmanienne: "Refaire le Timée », c'est-à-dire, non seulement tracer la logique interne du procès général de la nature dans tous ses degrés et toutes ses variations, mais également naturaliser l'idéel, en montrant « au sein des Idées elles-mêmes les raisons de son application à l'Univers sensible ». Dans une telle perspective, nous nous proposons d'étudier la manière dont deux pensées s'inscrivant dans la lignée de la philosophie de la Nature, le jeune Schelling d'un côté, Whitehead de l'autre, tentent d'élaborer les formes renouvelées de platonisme immanentisé, qui inscrirait l'idéel non comme objet mental ou transcendant, mais comme facteur intégralement naturel guidant le développement des êtres, sous la forme des " actions » physiques et organiques (Sur l'Âme du Monde, Esquisse d'un Système) chez Schelling, et celle des "objets éternels» (Procès et réalité), en nous intéressant notamment sur la manière dont ces concepts permettent d'offrir des réponses potentiellement novatrice dans le cadre de l'opposition traditionnelle du réalisme et du nominalisme, plus décisive à ce titre que le couple réalismeidéalisme, et sur l'implication de ces positions à l'égard de la question de la finalité au sein de la nature. 


\section{INDIVIDU ET COLLECTIF}

\section{Sebastian Kock - Max Stirner, C. B. Macpherson et la critique de la propriété}

Max Stirner (1808-1856) a été souvent considéré comme «la bête noire » dans la tradition de la philosophie politique en Allemagne. Le livre qui lui a accordé ce statut porte le titre Der Einzige und sein Eigenthum («L'Unique et sa propriété »), un texte consacré à l'élaboration d'une critique fondamentale de la propriété. Malgré les critiques rigoureuses de sa pensée (entre autres K. Marx, J. Habermas et H. G. Helms), Stirner, vir unius libri par excellence, n'a pas cessé de trouver des lecteurs des deux côtés du Rhin et on peut même observer un intérêt renouvelé pour la philosophie de Stirner en France. L'apparition de Stirner au programme d'agrégation d'allemand de la session 2017, ainsi qu'un colloque organisé par l'Université Paris 4 et l'Université de Lorraine à Paris en janvier de la même année en sont des indices. De la Déclaration des Droits de l'Homme et du Citoyen de 1789 en France en passant par le Grundgesetz de 1949 en Allemagne jusqu'à nos jours, la propriété fut interprétée dans les états libéraux en Europe comme un "principe fondateur» de la démocratie ou un "droit fondamental» de l'individu. En revanche, le politologue canadien C. B. Macpherson (1911-1987) y voit l'expression d'une culture de l'individualisme possessif dont il trace la généalogie jusqu'aux fondements de la pensée libérale (Hobbes, Locke). Dans mon exposé, j'essaye de combiner la théorie de l'individualisme possessif de Macpherson avec la critique de la propriété privée de Stirner pour en dégager des éléments qui pourraient servir à la construction d'un discours critique de la question du droit à la propriété dans le contexte actuel.

\section{Ludmilla LORRAIN - L'utilitarisme et l'idée d'Europe}

La doctrine utilitariste telle que Mill la formule énonce que le principe devant guider l'action politique est la promotion du plus grand bonheur pour le plus grand nombre d'individus possible. Ce principe le conduit, après Bentham, à poser qu'idéalement, ce sont les intérêts de l'humanité qui doivent être visés. Un tel principe devrait donc logiquement déboucher sur une forme de cosmopolitisme. Pourtant, au moment de décider quelle forme politique est la plus à même de réaliser ce principe, le choix de Mill, comme de Bentham, se porte sur la démocratie pure représentative : elle seule rend possible la mise en œuvre de l'impartialité exigée par le principe d'utilité stipulant que « chacun doit compter pour un, et aucun pour plus d'un». Et les textes que ces auteurs consacrent à la question de la démocratie représentative insistent sur le fait qu'une de ses conditions de réalisation est 
l'existence d'une communauté unie par des liens de sympathies, un certain ethos, rendant possible la constitution d'une opinion publique. Dès lors, ramené aux limites de l'État-nation, le principe d'utilité, dans extension maximale, semble condamné. Pourtant, la possibilité de cette extension du principe d'utilité n'est pas entièrement abandonnée par Mill : car si celui-ci rejette la possibilité d'institutions représentatives européennes, il soutient aussi, à plusieurs reprises, qu'une fédération des États européens serait le meilleur moyen d'arriver à la réalisation du principe d'utilité - et l'un des outils les plus efficace pour le progrès de l'humanité. Aussi entendons-nous rendre compte de cette ambivalence. Pour cela, il nous faudra, dans un premier temps, distinguer le constat d'une impossibilité liée à des contingences historiques, pouvant être dépassées, de l'idéal d'une fédération des États européens proposé par Mill. Cela nous amènera à préciser les raisons empêchant cette union au temps de Mill, ainsi qu'à comprendre pourquoi, par ailleurs, il considère l'Europe comme un espace privilégié - ce qui nous amènera à spécifier la notion d'ethos. Il nous faudra, enfin, reconstruire la forme que Mill donne à cette union possible. Alors seronsnous en mesure de montrer qu'il est possible, à la lumière de ces éléments, de dessiner l'idée d'une communauté de l'Europe unie par la notion d'intérêt commun.

\section{Nicolas LEMA HABASH - L'usage de la notion de vie spinoziste dans l'ouvre d'Alexandre Matheron}

Le travail d'Alexandre Matheron offre un des plus importants commentaires sur la philosophie de Spinoza. Dans cette intervention nous nous concentrons sur l'œuvre de Matheron en considérant ses mérites philosophiques propres. Cela nous oblige à faire un travail critique de son corpus afin de considérer comment en tant que philosophe son point de vue relève du commentaire. Plus précisément, nous voulons déterminer l'usage que Matheron fait de la notion spinoziste de « vie » dans ses ouvrages. Notre argument souligne que l'interprétation matheronienne de la notion de vie spinoziste lui permet d'établir une fonction "démocratique » autour de la possibilité d'arriver à la jouissance intellectuelle suprême. La possibilité d'une réactualisation du conatus dans la durée et la plasticité extrême du corps pour acquérir des nouvelles formes fournissent une théorie de la réincarnation humaine. Dans cette perspective, les êtres humains ont, non seulement une chance d'arriver à la jouissance suprême, mais en beaucoup. Dans la mesure où ses essences peuvent se réactualiser dans la durée potentiellement à l'infini, les êtres humains ont plusieurs vies pour arriver au fonctionnement optimal de ses conatus. C'est pourquoi nous dirons que Matheron utilise sa lecture de la notion de vie spinoziste pour fournir une interprétation politique démocratisant sur la possibilité d'arriver à la jouissance totale. Tous ont la possibilité d'y arriver, peut-être non dans cette vie, mais possiblement dans la prochaine, quand le contexte sera plus favorable pour cette démarche. 


\section{DROIT ET LOIS}

\section{Kyosuke TAHARA - Lois, mours et opinion publique chez Diderot}

Le vieux problème de la philosophie politique portant sur la relation entre lois et mœurs fut réactivé au siècle des Lumières, notamment par l'auteur de l'Esprit des lois. En accordant de l'importance au poids sociologique des conditions locales, Montesquieu a posé comme principe le non-empiètement entre lois et mœurs, sans pour autant nier un moyen non juridique de changer celles-ci. Or Rousseau a repris à son tour ce problème au profit du principe de souveraineté populaire dont l'application exige non seulement sa législation, mais aussi l'institution de mœurs dignes de cette constitution. Il a ainsi introduit le concept d'opinion publique comme une médiation entre lois et mœurs réglée plus ou moins par la censure qui veille à une cohésion sociale en incitant les citoyens à aimer leurs lois et à se dégager de la corruption. Face à cette mutation de la problématique $\mathrm{du}$ rapport entre lois, mœurs et opinion publique provoquée par ses contemporains, quelle position Diderot prend-t-il, lorsqu'il s'engage dans la réflexion politique au travers de ses écrits sur la Russie, de sa Lettre à Necker, et de sa collaboration à l'Histoire des deux Indes dans les années 1770 ? À l'inverse de Montesquieu, il affirme que, loin d'adapter la forme du gouvernement à la nature locale des choses, il est souhaitable de mettre en place partout, même dans un État despotique, la souveraineté $\mathrm{du}$ peuple, et de modifier les mœurs locales par la législation en fonction de cet impératif universel. Or contrairement à Rousseau, pour Diderot, l'institution de la souveraineté populaire ne renvoie pas au contrat social, ni à l'anticipation par le législateur de ce contrat. Il retient ici la leçon pluraliste de Montesquieu et impose plutôt de concevoir les diverses voies de ladite institution qui visent au même but, mais varient selon les modalités (réforme par en haut ou révolution populaire) et les conditions historiques (poids de l'ancienne législation et degré d'épuration ou de corruption des mœurs). Quant à l'opinion publique, bien qu'il admette sa corrélation avec les mœurs, Diderot lui assigne, non pas un moyen de régler les mœurs, mais une fonction de contre-force qui doit imprimer une direction de réforme du gouvernement en fonction de l'intérêt variable du peuple. Ainsi, en précisant une telle relation de causalité circulaire entre opinion publique, lois et mœurs chez Diderot, nous pourrons montrer comment il transpose certaines idées majeures de Montesquieu (importance des conditions locales et de la contreforce étatique) dans une perspective universaliste et réformatrice qui répond à l'aspiration démocratique. 


\section{Shingo Акімото - La réfutation de l'Empire universel et l'élaboration $d u$ ius gentium chez Jean Bodin}

À l'époque de la Renaissance, le juriste français Jean Bodin (1529/301596) a repris la conception de l'histoire universelle développée par Polybe pour créer un cadre d'étude adapté à la nouvelle réalité internationale de son époque: la mondialisation, à une échelle alors inédite. L'objet de l'intervention est de réfléchir sur cet usage de la conception polybienne de l'histoire universelle chez Bodin, que celui-ci oppose à une autre conception de l'histoire universelle fondée sur la théorie des quatre monarchies de Daniel (Da, 2, 1-49; 7, 1-28). Pour ce faire, il s'agit de s'interroger sur la place qu'occupe le chapitre VII de la Methodus ad facilem historiarum cognitionem (Méthode pour comprendre facilement les histoires) (1566 ; 1572), intitulé " Confutatio eorum, qui quatuor monarchias et aurea secula statuunt (Réfutation de ceux qui établissent les quatre monarchies et les siècles d'or)» dans son œuvre. La critique de Bodin vise surtout le Chronicon Carionis $(1558$; 1560), écrit par Johannes Carion (1499-1537) et augmenté et traduit en latin par Philippe Melanchthon (1497-1560), qui prépare la base de l'historiographie protestante. Dans cette œuvre, la théorie des quatre monarchies sert de principe structurel à l'historiographie de la translatio imperii, permettant de prétendre à l'universalité de l'histoire. Cette dernière est assurée par l'ordre chronologique de la translatio dans lequel un imperium unique est conservé par l'Empereur romain germanique. Une telle historiographie se heurte cependant à la notion d'histoire universelle polybienne reprise et remaniée par Bodin. Étant synchronique, celle-ci suppose la coexistence de la souveraineté (summum imperium) de chaque peuple dans le monde habité, et ainsi ne laisse aucun espace à l'existence d'un seul imperium romain. Elle est en même temps capable de fournir une vision synoptique pour la Respublica mundana (République mondiale) qui constitue le fondement du ius gentium (droit des gens), un composant du droit universel. L'histoire universelle et le droit universel sont ici considérés parallèlement. L'usage de la conception polybienne de l'histoire universelle amène donc, à nos yeux, à l'élaboration du ius gentium moderne.

\section{Fanny-Elisabeth ROLLET - Le rôle des émotions dans les excuses pénales}

Je me propose d'étudier les transformations du sens de la peine induites par les politiques pénales de la dangerosité, du temps de leur naissance dans un contexte de la lutte contre la récidive durant la seconde moitié du $19^{\mathrm{e}}$ siècle au moment de leur réactivation dans et par le droit pénal moderne. On prend ici pour exemple emblématique de cette renaissance, en France, la loi du 25 février 2008 relative à la rétention de sûreté et à la déclaration d'irresponsabilité pénale pour cause de trouble mental, sans toutefois limiter l'analyse à cette loi, et en tenant compte de l'ensemble législatif constitué par les lois «antiterroristes » en France depuis le milieu des années 1980, mais qui marquent un mouvement d'accélération dans 
la décennie 2010. Il s'agit ensuite - et surtout - d'en tirer toutes les conséquences sur le sujet de la peine et la fonction du droit pénal. De la triade italienne - Lombroso, Ferri et Garofalo - à la renaissance des thèmes de l'homme dangereux depuis le milieu des années 2000, en donnant une épure des implications de la logique préventive voire prédictive appliquée au droit pénal, on tentera de faire la synthèse des techniques d'imputation et d'individualisation propres aux politiques criminelles de dangerosité, techniques qui marquent de leur empreinte, dès sa naissance, la « raison criminologique ».

\section{Nicolas NAYFELD - Vaut-il mieux laisser un coupable en liberté que punir un innocent?}

En 1747, dans Zadig, Voltaire écrit qu'«il vaut mieux hasarder de sauver un coupable que de condamner un innocent $»$. Vingt ans plus tard, entre 1765 et 1769, sont publiés les Commentaires sur les lois anglaises de Blackstone, dans lesquels ce dernier écrit : « la Loi juge qu'il vaut mieux que dix coupables ne soient punis, qu'un innocent souffre le moindre dommage. » Le principe défendu par Voltaire et par Blackstone est qu'il est moralement pire de punir (intentionnellement ou sans l'avoir voulu) un innocent que d'acquitter (intentionnellement ou sans l'avoir voulu) un coupable. Ce principe a eu et continue d'avoir une influence majeure sur l'institution pénale et, en particulier, sur la procédure pénale, dans la mesure où la charge de la preuve incombe non pas à celui qui est accusé (devant prouver alors son innocence) mais à celui qui accuse (devant prouver alors la culpabilité de celui qu'il accuse). Toutefois, aussi intuitif soit-il, ce principe hiérarchisant les maux est problématique, d'un point de vue philosophique. Soient les deux justifications principales de la peine, le rétributivisme et l'utilitarisme. Si l'on est rétributiviste, aussi bien punir le coupable que ne pas punir l'innocent sont des impératifs catégoriques, d'importance égale : celui qui mérite d'être puni doit l'être, celui qui ne le mérite pas ne doit pas l'être. Si l'on est utilitariste, l'impunité d'un grand nombre de criminels est, tout bien considéré, peut-être un mal plus grand que la punition d'un petit nombre d'innocents. Ainsi, si le principe de Voltaire et Blackstone doit être défendu, sur quoi doit-il être fondé ? Peut-on le dériver du rétributivisme, de l'utilitarisme ou alors d'une théorie dite mixte? D'ailleurs, ce principe est-il si clair? Blackstone parle de dix coupables, mais pourquoi ce chiffre ? Pourquoi pas cent ou mille? Notre intervention sera l'occasion de revenir sur deux débats épineux de la philosophie pénale : celui, très connu, de la punition des innocents et celui, moins connu, de la non-punition des coupables. 